\title{
ARTICLE
}

\section{Does medium chain triglyceride play an ergogenic role in endurance exercise performance?}

\author{
Rodrigo Vitasovic Gomes ${ }^{1}$ and Marcelo Saldanha Aoki ${ }^{1,2}$
}

\begin{abstract}
Because of the medium chain triglycerides (MCT) specific physical and chemical properties, they have been used over the last 40 years in enteral and parenteral nutrition. Results from clinical practice lead some researchers in the early 80 's to use them for ergogenics purposes. The hypothesis was based on the relationship between the oxidation rates of carbohydrate and fat. The increase in fat oxidation would promote glycogen sparing effect, and therefore, delay the time to exhaustion. The aim of the present paper is to review the effects of MCT supplementation upon endurance exercise performance. Most of the studies failed to prove the ergogenic effect of MCT. A few studies that showed the ergogenic effect of MCT administration used alternative experimental designs, such as high MCT dose (above from the previous established limit) or infusion. The chronic use of MCT by athletes is new and few studies have been done in this matter. These few studies showed controversial results. There is a strong tendency in the literature that $\mathrm{MCT}$ is not a viable strategy to increase performance during endurance exercise. The aim of this study is to discuss the effects of MCT use on endurance exercise.
\end{abstract}

Key words: Medium chain triglycerides. Endurance. Ergogenic effect.

1. Laboratório de Fisiologia do Exercício - UniFMU, São Paulo, Brasil.

2. Instituto de Ciências Biomédicas - USP, São Paulo, Brasil.

Received in 10/10/02

$2^{\text {nd }}$ version received in $11 / 2 / 03$

Accepted in 10/3/03

Correspondance address:

Marcelo Saldanha Aoki

Instituto de Ciências Biomédicas - USP

Av. Lineu Prestes, 1.524, sala 410

05508-900 - São Paulo, SP

E-mail: msaoki@usp.br

\section{INTRODUCTION}

The progress of sports-related research over the past decades has added significantly to the increase of athletic performance. In regard to nutrition, research has typically focused on the relationship between the management of basic nutrients found in diet and performance enhancement ${ }^{1}$.

From this assumption came the idea of providing the athlete with a load of carbohydrate before the competition, to delay exhaustion. Overcompensation diet has successfully increased both muscular glycogen storage and performance ${ }^{2,3}$. It was thus established a direct relationship between muscular glycogen content and ability to perform exercises.

Based on these findings, from the 60 s, other nutritional strategies were developed to increase availability of free fatty acids (FFA) or fatty acids oxidation capability, both aiming to promote glycogen sparing effect and therefore delay exhaustion ${ }^{4}$.

Initially, studies by Rennie et al. ${ }^{5}$ and Hickson et al. ${ }^{6}$ used rats. In their studies, reduction of glycogen degradation rate and increased time for exhaustion after administration of corn oil were seen. These results encouraged the carrying out of tests on lipid supplementation in humans. Costill et al. ${ }^{7}$, Coyle et al. ${ }^{8}$, Dick et al. ${ }^{9}$ and Vukovich et al. ${ }^{10}$ showed that by increasing FFA concentration, it was possible for humans to reduce muscular glycogen utilization when performing endurance exercise. It has also been observed increase in exercise performance-maintenance capability.

It is important to mention that the techniques to raise FFA concentration employed in those studies include triglycerides intake followed by intravenous heparine infusion, but this is a too invasive procedure for a practical benefit of the athlete. Moreover, heparine is a powerful anti-coagulant, and could potentiate bleeding, in case of lesions.

\section{Medium chain triglycerides - MCT}

Due to their specific physical and chemical features, MCT have been used in enteral and parenteral diets for over 40 years ${ }^{11,12}$. They are made of fatty acids presenting 6 to 12 carbon atoms in their chain ${ }^{11-14}$. 
At room temperature, MCT is liquid; it is rapidly digested, and in the small intestine it splits into glycerol and 3 medium-chain fatty acid (MCFA) mollecules ${ }^{13,14}$. These compounds are submitted to lipase action at the gut and duodenum ${ }^{15}$.

Gastric emptying rate of a MCT solution is similar to the one of a carbohydrate isotonic beverage at rest ${ }^{16}$. MCFA absorption velocity is similar to the glucose one ${ }^{17}$. MCFA are more polar and hydrophilic than long-chain fatty acids (LCFA), therefore, they are more rapidly absorbed by the epitelial intestinal cells ${ }^{16}$.

Differently than LCFA, MCFA is not significantly incorporated in lipoproteins. MCFA is soluble in water ${ }^{13}$, and is transported via hilus hepatis to the liver, bound to albu$\min ^{14}$.

MCT has a swift oxidation at rest and under exercise, differently than the long-chain triglycerides $(\mathrm{LCT})^{18}$. LCFA intramitochondrial transport depends on carnitine ${ }^{19}$. MCFA, on the other hand, is transported through the mitochondrial membrane independently of the carnitine palmitoil-transferase (СРT) system ${ }^{20}$. Because of its features, described earlier, MCT is a swift source of power. However, due to the huge number of carbon atoms already bound to oxygen, and thus cannot be oxidated, MCT provides approximately 8 Kcal per gram ${ }^{11}$.

From the observation of the results presented by MCT, particularly in the medical practice, MCT intake prior of exercise has been recommended, in order to increase total lipid oxidation. The idea is that, by providing an alternative energetic substratum for extended, aerobic endurance exercises, carbohydrate endogenous supply would be less required $^{21-23}$.

\section{MCT CLINICAL APPLICATION}

MCT has been broadly used in clinical nutrition, when lipid digestion and absorption are compromised. Its use is a possible alternative to ensure proper supply of lipids and energy.

Among the conditions in which MCT should be used, one can mention lipid digestion disorders, due to lack of pancreatic lipases, or biliary atresia, or obstruction of bile duct, pancreatectomy or cystic fibrosis ${ }^{14,24,25}$, and absorptive disorders, such as Whipple disease, Crohn disease, and impaired neonatal absorption ${ }^{14,26,27}$. Clinical application include problems in lipid transportation, such as impaired kilomicron synthesis and lymphatic system disorders ${ }^{13,14}$.

Some clinical conditions, such as major burns and generalized infection, are marked by intense catabolism. Postoperative recovery patients also present increased protein catabolism. Lipidic emulsions with MCT are used in these cases to improve nitrogen balance and attenuate lean mass loss. Jiang et al. ${ }^{28}$ tested MCT and LCT supplementation in individuals under post-op recovery. In this study they observed higher nitrogen retention in the МCT supplement arm compared to the LCT arm. Such improvement in protein metabolism was related to an increase of serum ketonic bodies and insulin.

Some studies also related MCT intake to improvement of insuline-mediated glucose uptake. Eckel et al. ${ }^{29}$ submitted diabetic (non-insuline dependent) individuals to a diet rich in MCT, to observe its effect on glucose metabolism. In this study, the necessary amount of glucose to maintain serum glucose levels during constant insuline infusion was $30 \%$ higher in these individuals. It is also important to stress that Eckel et al. ${ }^{29}$ did not see changes in serum triglyceride concentration. These authors believe MCT intake is a promising strategy to treat type II diabetes, along with other conventional nutritional measures and administration of sulfonylureas.

Other possible MCT application is the treatment of individuals who present impaired synthesis of the enzyme that takes part in LCFA intramitochondrial transport. According to Parini et al. ${ }^{30}$, the use of MCT is a palliative strategy, to attenuate carnitine-acetylcarnitine translocase deficiency. MCT, however, cannot be considered an ideal source of power for patients with severe deficiency of this type of enzyme. Parini et al. ${ }^{30}$ further suggest that careful management of the diet can improve prognosis for this condition.

The appropriate diet during liver regeneration is still a puzzle. However, some investigations, such as the one by Blaha et al. ${ }^{31}$, assessed MCT effect on hepatic regeneration. After LCT and MCT supplementation during regeneration process, these authors ${ }^{31}$ concluded that a balanced MCT and LCT supplementation (40\% and 60\%) is the most appropriate, as it preserves liver regeneration, which was not observed with the use of only one, MCT or LCT.

A number of studies related the use of ketogenic diet to suppression of seizures in epileptic individuals ${ }^{32}$. MCT has also been studied in the treatment of convulsions, as partial MCFA oxidation in the liver favors the formation of ketonic bodies (aceto-acetate and beta-hydroxibutirate) $)^{33,34}$. Outcomes from the studies are yet controversial, particularly from animal models ${ }^{32}$. Thavendirathan et al..$^{35}$ observed that rats fed with a ketogenic diet (rich in MCT) that were further submitted to experimental models that stimulated convulsion did not present anti-convulsivant effect. In spite of the high serum levels of ketonic bodies those animals presented, Thavendirathan et al. ${ }^{35}$ suggest that this concentration does not necessarily suppress seizures.

MCT supplementation in some specific instances has been quite effective. These findings fostered a number of stud- 
ies, carried out in the 80 s, to test it in exercises ${ }^{21-23}$. However, it is not possible to assume that success in medical practice ensures effectiveness for physical exercises.

\section{MCT SUPPLEMENTATION IN ENDURANCE EXERCISES}

\section{Acute MCT administration}

Pioneer studies using MCT to enhance performance were carried out in the $80 \mathrm{~s}^{21-23}$. In the first 3 studies, no advantage on the use of MCT over carbohydrate was found.

A single study by Sabatin et al. ${ }^{36}$ demonstrated a positive effect from MCT supplementation. After intake of 45 grams of MCT, there was a proven reduction of glycogen oxidation rate $^{36}$.

Massicotte et al. ${ }^{37}$ have, afterward, compared intake of an MCT solution (approximately 30 grams) with one of glucose in humans before an extended exercise session (120 minutes), performed at $65 \%$ of $\mathrm{VO}_{2} \max$, in an ergometric cycle. Once oxidation rate of those substrata was assessed, it was demonstrated that both provided similar contribution for power generation during exercise. Both carbohydrate and MCT intake prevented glucose serum levels to fall, which typically occurs in longer-lasting exercises. Sustained serum glucose levels attenuated insuline reduction and increase of its regulatory counterparts (adrenaline and glucagon). According to these authors ${ }^{37}$, hormone adjustment would have made difficult the role of endogenous fatty acid in power generation, thus preventing glycogen sparing effect.

In Borghouts et al. ${ }^{38}$ study, a double-blind, crossed supplementation model was used to determine MCT effect on the utilization of muscle glycogen. It was noted that MCT supplementation did not differ from carbohydrate in glycogen content after 180 minutes of exercise at $61 \%$ of $\dot{\mathrm{V}} \mathrm{O}_{2}$ max in ergometric cycle.

Still in 1995, a study performed by Sidossis et al. ${ }^{39}$ achieved results that regarded interest on the use of MCT in exercise. These authors compared the effect of MCFA with LCFA infusion during an exercise of progressive intensity. This study showed that increase in intensity reduced LCFA and increased MCFA oxidation. In relation to MCFA metabolism, Jong-Yeon et al..$^{40}$ observed that endurance training enhances their oxidation.

After the above mentioned studies, the main focus of investigation was to compare intake of MCT alone, MCT associated to carbohydrates, and carbohydrates alone. Jeukendrup et al. ${ }^{41}$ gave MCT supplement to athletes, in order to raise their serum concentration of fatty acids and, therefore, enhance their oxidation. Results showed that during exercise performed in ergometric cycle at $57 \%$ of $\dot{\mathrm{V}}_{2} \max$ for 180 minutes, MCT oxidation rate, when associated to glucose, reached $72 \%$ of the intake amount. It was thus shown that MCT is an alternative source of energy for exercising. Due to low gastrointestinal tolerance, however, energetic contribution from MCT oxidation was insignificant (3\% to $7 \%$ of the energy used). Previous studies, such as that by Ivy et al. ${ }^{21}$, also confirmed that the maximum tolerance to MCT is 30 grams. In 1996, Jeukendrup et al..$^{42}$ induced glycogen depletion in humans and then administered MCT alone, MCT associated to carbohydrate or carbohydrate alone, prior to a 90 -minute exercise session at $57 \%$ of $\mathrm{V}_{2}$ max in ergometric cycle. It was then noted that reduction of stored glycogen exacerbated total fat oxidation ${ }^{42}$. However, oxidation of the administered MCT was not significantly increased. Once more, energy from MCT oxidation was insignificant.

In another study carried out by Jeukendrup et al. ${ }^{12}$, supplementation of 29 grams of MCT with carbohydrate during an exercise at $57 \%$ of $\dot{\mathrm{V}}_{2} \max$ for 180 minutes in ergometric cycle did not promote increase in the concentration of serum fatty acids, neither influenced the utilization of endogenous carbohydrate.

In 1996, a study carried out by VanZyl et al. ${ }^{43}$ reported performance improvement from MCT intake. The subjects were submitted to submaximal exercise $\left(60 \%\right.$ of $\dot{\mathrm{V}}_{2} \max$ for 120 minutes in ergometric cycle) followed by a an exercise test in cycloergometer in which one should traverse 40 kilometers in as short a time as possible. During exercise, athletes would receive, initially, $400 \mathrm{ml}$, followed by $100 \mathrm{ml}$ at every 10 minutes of one of the following solutions: MCT at $4.3 \%$ (MCT), MCT at $4.3 \%$ associated to carbohydrate at $10 \%(\mathrm{MCT}+\mathrm{CHO})$, or carbohydrate at $10 \%$ $(\mathrm{CHO})$. Athletes who received $\mathrm{MCT}+\mathrm{CHO}$ traversed the 40 kilometers in significantly less time than the others. There was no difference in serum glucose levels, glycerol and endogenous fatty acids between $\mathrm{MCT}+\mathrm{CHO}$ group and $\mathrm{CHO}$ only group ${ }^{43}$.

For the first two hours, total serum fatty acid concentration was unchanged. Only from the third hour of the test on, the MCT + CHO group presented increase in MCFA and ketonic bodies concentration. At the same time, this group presented reduced lactate production compared to $\mathrm{CHO}$ group. These results lead to the conclusion that reduced oxidation of endogenous carbohydrate in the MCT group was due to ketogenesis increase ${ }^{43}$.

The amount of MCT used by VanZyl et al. ${ }^{43}$ (86 grams) was significantly higher than the amount given in prior studies. These authors propose that the reduced MCT amount, of approximately 30 grams $(4 \mathrm{ml} / \mathrm{kg}$ in the beginning, and $2 \mathrm{ml} / \mathrm{kg}$ at every 20 minutes, of a solution at $5 \%$ ), used in prior studies ${ }^{12,41,42}$ was not effective in promoting increase 
of serum fatty acids and, therefore, glycogen sparing effect.

The findings of VanZyl et al. ${ }^{43}$ caused a lot of controversy among investigators of the field as, up to now, studies had respected the intake limit of 30 grams of MCT. Therefore, to corroborate these findings, Jeukendrup et al. ${ }^{44}$ carried out a study similar to VanZyl's et al. ${ }^{43}$, supplementing subjects with 85 grams of MCT. Subjects exercised for 120 minutes at $60 \%$ of $\dot{\mathrm{V}}_{2}$ max, immediately followed by a cycloergometer testing. Administration of solutions was done as follows: $8 \mathrm{ml} / \mathrm{kg}$ at the beginning of the exercise, and 2 $\mathrm{ml} / \mathrm{kg}$ at every 15 minutes during exercise. There was no significant differences in the time taken to perform exercise between carbohydrate solution at $10 \%$ and MCT at 5\% + carbohydrate at $10 \%$ solution. MCT at $5 \%$ solution not only cause gastrointestinal discomfort, but impaired performance, compared to placebo (water) and carbohydrate at $10 \%$ solution groups.

That controversy on the effect of the MCT amount on performance lead to a new study. Goedecke et al. ${ }^{45}$ gave 9 cyclists one of three supplements: $10 \%$ glucose solution, $10 \%$ glucose solution $+1,72 \%$ MCT, or $10 \%$ glucose + $3.44 \%$ MCT. The investigators were also interested in studying the effects of progressive MCT intake on gastrointestinal tract and carbohydrate oxidation during cycloergometer exercise with 120 -minute duration at $63 \%$ of $\mathrm{V}_{2}$ max.

FFA and ketonic bodies concentration raised, but this did not affect oxidation of this substratum nor cyclists performance. The high carbohydrate intake (approximately 140 grams) before the exercise, in that study, raised the level of serum insulin. According to Goedecke et al..$^{45}$, this facilitated carbohydrate, rather than fatty acid oxidation, in spite of the high concentration of the later after MCT intake.

In Van Zyl et al. study ${ }^{43}$, the meal prior to the test included 85 grams of carbohydrate. The amount of carbohydrate intake resulted in serum insulin concentration close to 6-7 $\mathrm{mU} / \mathrm{L}$, whereas in Goedecke et al. ${ }^{45}$ study, serum insuline concentration ranged between 13-24 mU/L. To Goedecke et al. ${ }^{45}$, ergogenic benefit from MCT may be limited by the increase of serum insuline and its inhibition effect on lipolysis and fatty acid oxidation.

As to gastrointestinal discomfort, Goedecke et al. ${ }^{45} \mathrm{ob}-$ served that only 2 athletes were intolerant to MCT. Jeukendrup et al. ${ }^{44}$, conversely, suggested that lack of performance improvement in their investigation could be partially due to gastrointestinal discomfort, also reported by Ivy et al. ${ }^{21}$ and Decombaz et al. ${ }^{22}$. To Goedecke et al. ${ }^{45}$, MCT tolerance is an individual feature, and cannot be considered as the sole limiting factor to performance. Those results suggest that athletes should test their tolerance to MCT at training sessions, before using it at a competition.
The main purpose of Horowitz et al. study ${ }^{46}$ was to investigate if a tolerable intake of medium-chain triglycerides (25 grams) actually decreases muscle glycogenolysis and muscular glycogen oxidation during intense exercise ( $84 \%$ of $\mathrm{VO}_{2}$ max). Their subjects were 7 well-trained cyclists who should perform high-intensity exercise for 30 minutes on cycloergometer, being the first 2 minutes at $60 \%$ of $\dot{\mathrm{V}}_{2}$ max and the ensuing 28 minutes at $84 \%$ of $\dot{\mathrm{V}}_{2} \max$, 60 minutes after carbohydrate intake $(0.72$ grams of sucrose $/ \mathrm{kg}$ ) alone or associated to MCT (0.36 gram of MCT/ $\mathrm{kg}+0,72$ gram of sucrose $/ \mathrm{kg}$ ).

Horowitz et al. ${ }^{46}$ reported that serum FFA did not increase, but this does not mean that ingested MCT did not enter blood stream. As Massicotte et al. ${ }^{37}$ previously showed, about $60 \%$ of a MCT meal was oxidated during exercise, without increase of serum FFA higher than in controls. In this study, MCT supplementation did not affect substratum oxidation during high-intensity exercise.

According to Angus et al. ${ }^{47}$, most studies assess MCT supplementation effects from exercises of constant and moderate intensity. For them, however, these experimental models do not replicate the conditions of an actual competition. In order to simulate a competition environment, a cycloergometer test was carried out, in which 100 kilometers should be traversed in as little time as possible. Three different solutions - carbohydrate $(6 \%)$, carbohydrate $(6 \%)$ + MCT $(4.3 \%)$, and sweet placebo - were administered as follows: $250 \mathrm{ml}$ of solution before the test and $250 \mathrm{ml}$ during exercise, at 15-minute intervals until the end. Angus et al. ${ }^{47}$ noted that athletes kept a similar level of work generation (watts) for the first 135 minutes, regardless of what they drank, which decreased close to the end of the exercise for the placebo group, only.

Angus et al. ${ }^{47}$ also showed that $\mathrm{CHO}$ intake over $100 \mathrm{ki}-$ lometers by well-trained athletes enhanced performance. The adding of MCT to CHO beverage did not change FFA serum concentration nor promoted further reduction in the stored $\mathrm{CHO}$, compared to $\mathrm{CHO}$ alone. The suggested explanation was that high intensity exercise decreases blood flow to the gastrointestinal tracts, affecting MCFA serum availability and thus limiting its energetic contribution.

As to acute MCT consumption to preserve muscular glycogen, most studies failed to show positive results. Among the few studies ${ }^{36,39,43}$ that demonstrated MCT ergogenic effect, some aspects should be stressed. In Sidossis et al. study ${ }^{39}$, MCT was intravenous admnistered. After being absorbed, MCFA follow through the hilus hepatis directly to the liver, where they are rapidly metabolized. Intravenous administration is, therefore, a strategy to overcome the physiologic barrier that liver represents for these fatty 
acids, and this would increase their availability to the muscle.

Both in Sabatin et al..$^{36}$ and in VanZyl et al. ${ }^{43}$ studies, the amount of MCT administered is higher than the gastrointestinal tolerance limit proposed as safe by the literature. In VanZyl et al. ${ }^{43}$ study, carbohydrate intake prior to the exercise was lower than in other studies. This has influenced insulin concentration, adding to a higher FFA mobilization and oxidation. The amount of ingested carbohydrate may have also contributed for discrepant results. Such differences in experimental models prevent direct outcomes comparison.

As to acute MCT supplementation, the challenges one still has to face are establishing maximum MCT tolerance, and the confirmation of a dose-dependent relationship between MCT and glycogen sparing effect.

\section{Chronic MCT administration}

One of the pioneer studies to assess chronic use of MCT was Fushiki's et al. ${ }^{48}$. Its aim was to investigate the effect of chronic MCT intake (6 weeks) in swimming endurance capability of sedentary and trained rats. Two diets were prepared, one with 80 grams of MCT +20 grams of LCT, the other with 100 grams of LCT.

Swimming capability for groups receiving chronic MCT supplements was the same as for the LCT supplement group. However, two weeks after the beginning of training associated to supplementation, MCT group presented a significantly higher resistance to fatigue than the LCT group ${ }^{48}$. Resistance to fatigue of rats that received a single MCT dose (acute supplementation) was not affected ${ }^{48}$. These data confirmed most results from acute MCT intake studies.

Fushiki et al ${ }^{48}$ further observed that sedentary and trained rats fed with МСT for 6 weeks took longer for exhaustion. For these authors, increase in concentration of ketonic bodies and fatty acids from the chronic MCT intake may have partially added to performance improvement.

In a study we carried out ${ }^{49}$, chronic supplementation (8 weeks) with palmiste oil (rich in medium-chain fatty acids - C12:0) muscle glycogen sparing effect was evident, compared to the control group. However, at the end of the treadmill workout, carried out for 60 minutes at $65 \%$ of maximum oxygen uptake ( $\left.\dot{\mathrm{V}}_{2} \max \right)$, liver glycogen levels were decreased, compared to controls ${ }^{49}$. It is to be stressed that, in rodents, hypoglycemia-related liver glycogen depletion precedes muscular glycogen reduction ${ }^{50}$. Therefore, for rats, liver glycogen is a limiting factor for exercise performance. In spite of time for exhaustion not having been assessed, we believe reduction of the liver glycogen storage is deleterious for exercise maintenance capability ${ }^{49}$.
Studies in humans, such as Goedecke's et al. ${ }^{51}$ and Missel's et al. ${ }^{52}$, as well as those in rodents, present controversial outcomes. Goedecke et al. ${ }^{51}$ investigated the effect of an isocaloric diet, rich in MCT, for 15 days. Each experiment included 150 minutes on cycloergometer at $70 \%$ of $\dot{\mathrm{VO}}_{2 \text { peak }}$, immediately followed by a test in which 40 kilometers should be traversed in as little time as possible. Before the experiment, athletes ingested an emulsion of orange flavored $10 \%$ carbohydrate and $3.44 \%$ of MCT. In this study, chronic intake associated to acute ingestion of MCT was assessed.

Goedecke et al. ${ }^{51}$ reported that exposure to a hyperlipidic diet for a short period of time, such as 5 to 10 days, leads to an increase in fat oxidation and, at the same time, spares muscular glycogen during exercise, compared to the control diet. This change in the metabolism of the substratum may occur due to increase of the CPT enzyme complex in the muscle and to the low muscular glycogen stored. However, no reduction in the time to traverse 40 kilometers was seen.

In Missel et al..$^{52}$ study, the aim was to determine the effect of chronic MCT intake on endurance exercise performance of runners. Participants ingested 56 grams of LCT and 60 grams of MCT per solution, daily, for two weeks. This recommendation was followed until the day before the test, keeping total fat intake of approximately $30 \%$ of the total power. The test was performed on a treadmill, at $85 \%$ of $\mathrm{VO}_{2} \max$ for 30 minutes, after which intensity was reduced to $75 \%$ of $\mathrm{VO}_{2}$ max until voluntary exhaustion. MCT supplementation did not cause time to exhaustion to increase, compared to LCT. Initially, MCT intake caused discomfort in all participants; however, after 2 weeks, most participants reported symptom decrease. Missel et al. ${ }^{52}$ believe chronic MCT intake increases tolerance to its acute ingestion, and that safety limit of 30 grams, previously suggested, may be changed.

In spite of some promissing results from the chronic use of MCT, it is to be stressed that there are few studies that assessed side effects from this practice. We noted that chronic and high intake of palmiste oil (rich in lauric acid C12:0) in sedentary rats triggered deleterious adaptations, such as GI index reduction (glucose and insulin ratio, broadly used to assess peripheral resistance to insulin), increase of serum triglycerides and proportion of fat ${ }^{53-55}$. In trained rats, however, such deleterious effects from chronic MCT use were attenuated ${ }^{55}$.

Chronic MCT administration to humans, to enhance physical performance, is recent and little explored. Some issues are still open, such as the ideal duration of supplementation period, and the proportion of lipids in relation to the overall calories ingested. Only after solving these 
issues, it will be possible to conclude if MCT provides ergogenic benefit with no health hazards.

\section{CONCLUSIONS}

Over the past 20 years, MCT use for endurance exercises was the focus of a number of investigations. However, some issues are yet to be solved in regard to MCT supplementation, such as assessing if glycogen sparing effect has a dosedependent relationship with MCT administration, and to determine if gastrointestinal tolerance is an individual feature. As to the safe limit for intake, more evidences are necessary to confirm this can be changed by chronic MCT use.

\section{REFERENCES}

1. Sá CA, Portela LOC. Manipulação de carboidratos na dieta e o diagnóstico da performance. Rev Bras Cien Mov 2001;9:13-24.

2. Alhborg B, Bergstrom J, Ekelund LG, Hultman E. Muscle glycogen and muscle electrolytes during prolonged physical exercise. Acta Physiol Scand 1967;70:129-42.

3. Bergstrom J, Hermansen L, Hultman E, Saltin B. Diet, muscle glycogen and physical performance. Acta Physiol Scand 1967;71:140-50.

4. Aoki MS, Seelaender MCL. Suplementação lipídica para atividades de endurance. Rev Paul Educ Fis 1999;13:230-6.

5. Rennie MJ, Winder WW, Holloszy JO. A sparing effect of increased plasma fatty acids on muscle and liver glycogen content in the exercising rat. Biochem $\mathrm{J}$ 1976;156:647-55.

6. Hickson RD, Rennie MJ, Conlee RK, Winder WW, Holloszy JO. Effects of increased plasma fatty acids on glycogen utilization and endurance. J Appl Physiol 1977;43:829-33.

7. Costill DL, Coyle E, Dalsky G, Evans W, Fink W, Hoopes D. Effects of elevated plasma FFA and insulin on muscle glycogen usage during exercise. J Appl Physiol 1977;43:695-9.

8. Coyle EF, Coggan AR, Hemmert MK, Fink W, Hoopes D. Muscle glycogen utilization during prolonged strenuous exercise when fed carbohydrate. J Appl Physiol 1986;61:164-72.

9. Dick DJ, Putman GJF, Heigenhauser E, Hultman E, Spriet LL. Regulation of fat-carbohydrate interaction in skeletal muscle during intense aerobic cycling. Am J Physiol 1983;265:E852-9.

10. Vukovich MD, Costill MS, Hickey SW, Trappe KJ, Fink WJ. Effect of fat emulsion infusion and fat feeding on muscle glycogen utilization during cycling exercise. J Appl Physiol 1993;75:1513-8.

11. Berning JR. The role of medium-chain triglycerides in exercise. Int $\mathbf{J}$ Sport Nutr 1996;6:121-33.

12. Jeukendrup AE, Saris WHM, Diesen RV, Brouns F, Wagenmakers AJM. Effect of endogenous carbohydrate availability on oral medium-chain triglyceride oxidation during prolonged exercise. J Appl Physiol 1996; 80:949-54.

13. Babayan VK. Medium-chain triglycerides - Their composition, preparation and application. J Am Oil Chem 1968;45:23-5.

14. Bach AC, Babayan VK. Medium-chain triglycerides: an update. Am J Clin Nutr 1982;36:950-62.

15. Galobert C, Filliat M, Chazalette JP, Mendy F, Delhaye M. Intestinal absorption of medium-chain triglycerides in a case of pancreatic cystic fibrosis. Ann Pediatric 1975;22:745-53.
As to the question posed in the title of this review, one may conclude that there are some controversies about MCTinduced performance. Currently, however, there is a strong tendency in the literature not to associate MCT supplementation to glycogen sparing effect and performance enhancement. Except for some few studies that suggested ergogenic benefit from MCT intake, most results show this diet strategy is poorly effective, and is not superior to carbohydrate supplementation.

All the authors declared there is not any potential conflict of interests regarding this article.
16. Beckers EJ, Jeukendrup AE, Brouns F, Wagenmakers AJM, Saris WHM. Gastric emptying of carbohydrate-medium chain triglycerides suspensions at rest. Int J Sports Med 1992;13:581-4.

17. Ashbrook JD, Spector AA, Fletcher JE. Medium-chain fatty acid binding to human plasma albumin. J Biol Chem 1972;247:703-8.

18. Metges CC, Wolfram G. Medium and long-chain triglycerides labeled with $\mathrm{C}$ : comparison of oxidation after oral or parenteral administration in humans. J Nutr 1991;121:31-6.

19. Fritz IB, Yue KTN. Long chain carnitine acyl transferase and the role of acylcarnitine derivates in the catalytic increase of fatty acid oxidation induced by carnitine. J Lipid Res 1963;4:279-88.

20. Bremer J. Carnitine and its role in fatty acid metabolism. Trends Biochem Sci 1980;2:207-9.

21. Ivy JL, Costill DL, Fink WJ, Maglisho E. Contribution of medium and long triglycerides intake to energy metabolism during prolonged exercise. Int J Sports Med 1980;1:15-20.

22. Decombaz J, Arnaud MJ, Milon H, Moesh G, Philopossian AL, Thelin $\mathrm{H}$, et al. Energy metabolism of medium-chain triglycerides versus carbohydrates during exercise. Eur J Appl Physiol 1983;52:9-14.

23. Auclair E, Satabin P, Servan E, Guezennec CV. Metabolic effects of glucose medium chain triglycerides and long chain triglycerides feedings before prolonged exercise in rats. J Appl Physiol 1998;57:126-31.

24. Gottschlich MM. Selection of optimal lipid sources in enteral and parenteral nutrition. Nutr Clin Pract 1992;7:152-65.

25. Valdivieso V. Absorption of medium-chain triglycerides in animals with pancreatic atrophy. Am J Digest Dis 1972;17:129-36.

26. Hofmann AF, Poley JR. Role of bile acid mal absorption in pathogenesis of diarrhea and steatorrhea in patients with ileal resection. Gastroenterology 1972;62:918-34.

27. Tantibhedhyangkul P, Hashim SA. MCT feedings in premature infants: effects of calcium and magnesium absortion. Pediatrics 1978;61:53745 .

28. Jiang Z, Zhang S, Wang X. Metabolic effects of medium-chain triglycerides in parenteral nutrition after surgery. Zhonghua Wai Ke Za Zhi 2001;39:694-7.

29. Eckel RH, Hanson AS, Chen AY, Berman JN, Yost TJ, Brass EP. Dietary substitution of medium chain triglycerides improves insulin-mediated glucose metabolism in NIDDM subjects. Diabetes 1992;41:641-7.

30. Parini R, Invernizzi F, Menni F, Garaveglia B, Melotti D, Rimoldi M, et al. Medium-chain triglycerides loading test in carnitine-acylcarnitine 
translocase deficiency: insights on treatment. J Inherit Metab Dis 1999; 22:733-9.

31. Blaha V, Cervinkova Z, Simek J, Cotkova H, Zaoak Z. Long and medium-chain triacylglycerols in nutritional support of liver regeneration of partially hepatectomized rats. J Physiol Res 1999;48:457-63.

32. Colleone VV. Aplicações clínicas dos ácidos graxos de cadeia média. In: Curi R, Pompéia C, Miyasaka CK, Procopio J, editores. Entendendo a gordura: ácidos graxos. 1a ed. Barueri: Manole, 2002;199-214.

33. Bach A. Oxaloacetate deficiency in MCT-induced ketogenesis. Arch Int Physiol Biochem 1978; 86:1133-42.

34. Bach A, Debry G, Metais P. Hepatic metabolism of medium-chain triglycerides. Bibl Nutr Dieta 1977;25:24-35.

35. Thavendiranathan P, Mendonca A, Dell C, Likhodii SS, Musa K, Iracleous $\mathrm{C}$, et al. The MCT ketogenic diet: effects on animal seizure models. Exp Neurol 2000;161:696-703.

36. Sabatin P, Portero GD, Bricout J, Guezennec CY. Metabolic and hormonal responses to lipid and carbohydrate diets during exercise in man. Med Sci Sports Exerc 1987;19:218-23.

37. Massicotte D, Peronnet F, Brisson GR, Hillaire-Marcel C. Oxidation of exogenous medium-chain free fatty acids during prolonged exercise: comparison with glucose. J Appl Physiol 1992;73:1334-9.

38. Borghouts AE, Juekendrup AE, Saris WHM, Brouns F, Wagenmakers AJM. No effect of medium chain triglycerides (MCT) ingestion during prolonged exercise on muscle glicogen utilization. Med Sci Sports Exerc 1995;27:S101-5.

39. Sidossis LS, Gastaldelli A, Wolfe RR. Fatty acid uptake by the mitochondria limits fat oxidation in strenuous exercise. Med Sports Sci Exerc 1995;27:S102.

40. Jong-Yeon K, Hickner RC, Dohm GL, Houmard JA. Long and medium chain fatty acid oxidation is increased in exercise trained human skeletal muscle. Metabolism 2002;51:460-4

41. Jeukendrup AE, Saris WHM, Schrauwen P, Brouns F, Wagenmakers AJM Metabolic availability of medium-chain triglycerides coingested with carbohydrates during prolonged exercise. J Appl Physiol 1995;79:75662.

42. Jeukendrup AE, Saris WHM, Brouns F, Halliday D, Wagenmakers AJM Effects of carbohydrate and fat supplementation on carbohydrate metabolism during prolonged exercise. Metabolism 1996;45:915-21.

43. Van Zyl CG, Lambert EV, Hawley JA, Noakes TD, Dennis SC. Effects of medium-chain triglyceride ingestion on fuel metabolism and cycling performance. J Appl Physiol 1996;80:2217-25.
44. Jeukendrup AE, Thielen JJHC, Wagenmakers AJM, Brouns F, Saris WHM. Effect of medium-chain triacylglycerol and carbohydrate ingestion during exercise on substrate utilization and subsequent cycling performance. Am J Clin Nutr 1998;67:397-404.

45. Goedecke JH, Elmer-English R, Dennis SC, Schoss I, Noakes TD, Lambert EV. Effects of medium-chain triacylglicerol ingested with carbohydrate on metabolism and exercise performance. Int J Sport Nutr 1999;9: 35-47.

46. Horowitz JF, Mora-Rodriguez R, Byerley LO, Coyle, EF. Pre-exercise medium-chain triglyceride ingestion does not alter muscle glycogen use during exercise. J Appl Physiol 2000;88:219-25.

47. Angus JD, Hargreaves M, Dancey J, Febbraio MA. Effect of carbohydrate or carbohydrate plus medium-chain trygliceride ingestion on cycling time trial performance. J Appl Physiol 2000;88:113-9.

48. Fushiki T, Matsumoto K, Inoue K, Kawada T, Sugimoto E. Swimming endurance capacity of mice is increased by chronic consumption of medium-chain triglycerides. J Appl Physiol 1995;57:126-31.

49. Aoki MS, Belmonte MA, Seelaender MCL. Effect of high fat diets and training upon muscle carnitine palmitoyltransferase activity and glycogen content. Med Sci Sports Exerc 2001;33:S213.

50. Terjung RL, Baldwin KM, Winder WW, Holloszy JO. Glycogen repletion in different types of muscle and liver after exhausting exercise. Am J Physiol 1973;226:1387-91.

51. Goedecke JH, Christie C, Wilson G, Denis SC, Noakes TD, Hopkins WG, et al. Metabolic adaptations to a high-fat diet in endurance cyclists. Metabolism 1999;48:1509-17.

52. Misell LM, Lagomarcino ND, Schuster V, Kern M. Chronic mediumchain triacylglicerol consumption and endurance performance in trained runners. J Sports Med Phys Fitness 2001;41:210-5.

53. Aoki MS, Belmonte MA, Seelaender MCL. Efeito da suplementação lipídica sobre o percentual de gordura da carcaça e de diversos tecidos de ratos Wistar. Anais da XIV Reunião da Fesbe 1999;383-4.

54. Aoki MS, Belmonte MA, Seelaender MCL. The partitioning of liver triacylglycerol pool is altered by high fat diets. Anais da XV Reunião da Fesbe 2000;73.

55. Aoki MS. Efeito da suplementação lipídica sobre parâmetros metabólicos de ratos submetidos ao treinamento de endurance [Dissertação apresentada para obtenção do título de mestre em ciências]. São Paulo: Instituto de Ciências Biomédicas da Universidade de São Paulo; 2000. 